\title{
CFD Simulation of Effect of Vortex Ring for Squid Jet Propulsion And Expeiments on a Bionic Jet Propulsor
}

\author{
Li Jian $^{1}$, Zhao Jianing ${ }^{1}$ and Wang Zhenlong ${ }^{2}$ \\ ${ }^{1}$ Northeast Forestry University, Harbin 150040, China \\ ${ }^{2}$ School of Mechatronics Engineering, Harbin Institute of Technology, Harbin \\ 150001, China \\ Lijian499@163.com
}

\begin{abstract}
Using jet propulsion, squid can swim at high speed or at low speed with good maneuverability, which makes them quiet valuable to be studied for biomimetic purposes. Vortex rings usually occur in the highly-unsteady jet flow in squid, and they play quite important roles in the jet propulsion of squid. This paper tries to investigate the squid jet structure by computational fluid dynamics (CFD) analysis. A simplified squid body model was established. The mantle motion during jet locomotion was explicitly included into the simulations by using a deforming mesh. By solving the $2 D$-axisymmetric, incompressible, laminar, unsteady Navier-Stokes equations, different vortex evolution behaviors were observed depending on different mantle contraction velocities and nozzle diameters. An important parameter, the formation number of the vortex rings, L/D, which decide the propulsive efficiency of jet propulsion directly, was also discussed in this paper. The numerical results show that adult squid propel themselves by long jet flows with a large formation number, L/D. The results also prove that smaller squid have larger relative funnel diameter. Interaction of vortex rings was simulated in two jet process, which might interpret squid increase their contraction frequencies with elevated swimming speed. To validate the force generated in the simulation, a bionc squid mantel jet propulsor is investigated and tested.
\end{abstract}

Keywords: Squid, CFD Simulation, Jet propulsion, Vortex rings, bionic jet propulsor

\section{Introduction}

Squid are among the most versatile aquatic swimmers, while they are also unusual swimmers since the thrust is generated by fin undulating and jet propulsion. The locomotion of squid is also a result of fast, efficient and adjustable movement of millions of years of evolutionary optimization. Squid not only can keep low energy expense, high propulsive efficiency and high maneuverability at low speed but also can realize quite high speed. The jet propulsion is the foundation of a squid's locomotive system. Squid are able to propel themselves in various directions by muscularly directing their funnel. Squid swim with high jet thrust, low jet frequency and low noise. Squid-inspired design could mean better handling of underwater vehicles, so research squid movement in water is a bionic fun and challenging areas of research.

While squid is the most versatile swimmer, relies on two locomotor system, little is known about the mechanical properties of squid swimming. Erik J. Anderson used a high-speed digital video to record squid swimming, to accurately determine its movement parameters, physical deformation and mantle diameter. Jet velocity jet thrust and chamber pressure are calculated by the video data.They observed that adult long-finned squid which are about $27 \mathrm{~cm}$ in length have a $9-32 L / D$ ratio [1]. Joseph T. Thompson and William M. Kier investigated the kinematics of mantle 
movement during escape jet behavior and focused on the relationship between ontogenetic changes in the kinematics of the mantle and the thrust generated during the escape jet. They measured the peak thrust and the kinematics of the mantle of squid ranged in size from 5 to $40 \mathrm{~mm}$ tethered to a force transducer [2-3]. Houshuo Jiang and Mak A. Grosenbaugh set up a numerical model to simulate squid jet propulsion. Object numerical simulation of a symmetrical body having a round shaft opening, with internal piston, the piston movement of water discharged through the use of a deformable mesh simulated movement of the piston. According to the ratio of different backgrounds and piston velocity (U/Up)and the ratio of the speed of the piston stroke and the opening diameter( $\mathrm{Lm} / \mathrm{D})$, observe the evolution of the vortex behavior. The basic principle is that the interaction between the jet and the starting vortex layer between the layers[4].

Usually squid would die as soon as they are captured from the ocean, even if they were live, they may not be able to swim as well as in the vast ocean, so direct observation of squid in oceans need complicated experimental equipments such as underwater vehicle and underwater camera which will result in high expense and long period. Consequently, it is difficult to do experiments on live squid. As the development of computer technology, computational fluid dynamics (CFD) analysis has been applied to understanding the mechanism of swimming fish successfully. This method not only can save money and time, but also can obtain comprehensive experimental data. In the present study, a CFD simulation of $2 \mathrm{D}$-axisymmetric simplified squid jet model has been developed to investigate the well-developed jet flow in swimming squid. The mantle motion during escape-jet locomotion was explicitly included into the simulations by using a deforming mesh. A parametric analysis of variables which affect vortex evolution behavior is presented, associated with flow visualization.

\section{Principle of Jet Propulsion of Squid}

Squid is a software marine life, are cephalopods, use jet high-speed mode and fin low speed mode to swimming. Figure 1 shows the injection process of squid. During jet propulsion, mantle first swell, causing water into the mantle cavity through the sides of the head and mantle. Then close the mantle, mantle body pressure chamber increases, the end of the absorption process. Then open funnel, water flows under pressure funnel, the thrust based on the principle of conservation of momentum provided by the high-speed movement can push squid.

The mantle musculature which functions as a constant volume system called a muscular-hydrostat consists of two muscle types: circular muscles and radial muscles. During jet propulsion, the contraction of radial muscles causes the mantle wall to become thinner. Since the mantle volume and the mantle length almost remain the same, the mantle diameter increaces, the diameter of the mantle must increase. The result is that water is sucked into the mantle cavity. Then circular muscles within the mantle contract, which consequently decreases mantle diameter and forces intramantle water out of the funnel. Accordingly, contraction of circular muscle results in concomitant thickening of the mantle wall. Especially, the radial muscles may not act when the mantle refill slowly [5]. 


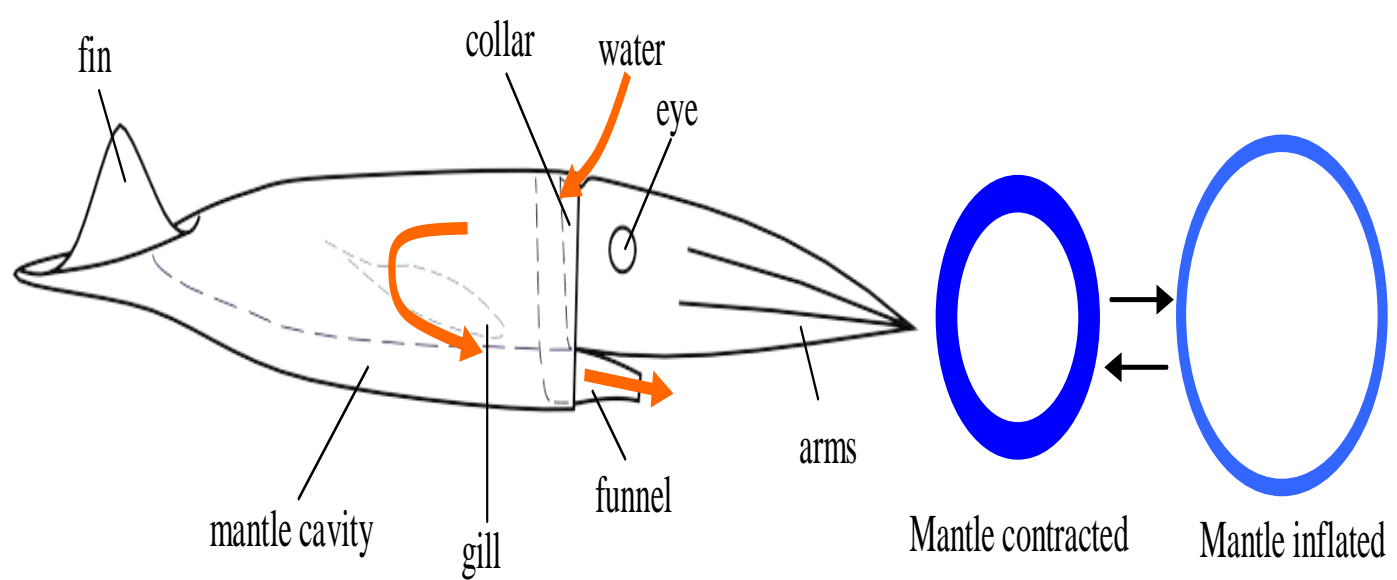

Figure 1. Scheme of Jet Process for Squid

\section{Effect of Vortex Ring}

For decades, the vortex ring attracted the attention of researchers, because they are a significant phenomenon of aquatic life swim bell. Shariff and Leonard made a comprehensive analysis of the ring vortex research experiments and numerical simulation use, including the formation of vortex rings, evolution and interaction[6].Observations of swimming fishes and other organisms such as salps or squids reveal a series of vortex rings forming behind the animals, which play an important part in their mechanisms of propulsion. Fishes produce these vortices by an undulatory motion of the body and tail, controlling the periodic shedding of vorticity into the wake [7], and salps or squids form them more directly, by ejecting fluid backwards through an orifice [8-9]. In both cases the vortices roll up into three-dimensional (3D) ring-like structures [10-11]. Laboratory experiments have demonstrated that the vortex rings make a proportionally larger contribution to mass and momentum transport than an equivalent straight jet of fluid. Previous researches have shown that vortex rings generated in still water in the laboratory can be optimized for efficiency or thrust, based on the jet length-to-diameter ratio $(L / D)$, with a maximum single leading vortex ring formation and peak performance occurring at $3.5<L / D<4.5[12-13]$. It shows that these animals can produce optimal vortex rings to achieve the maximum thrust for a given energy input.

Gharib et al. [14-16] observed that the formation of vortex rings in jets emitted from cylindrical pipes into still water was dependent upon the ratio of the length of the plug of fluid expelled from the pipe, $L$, and the inside diameter of the pipe, D. The ratio $L / D$ can be called a formation number for vortex ring. When the $L / D$ ratio is less than approximately 4, a solitary vortex ring was formed and no other flow structure was present, that means all the vorticity shed from the pipe was bound up in this single vortex ring. The intensity of the vortex ring is getting stronger as the increasing of the $L / D$ ratio in the range of $0-4$. When the $L / D$ ratio approximately equals 4 , the intensity of the vortex ring achieves max. When the $L / D$ ratio is greater than approximately 4, the vorticity shed from the pipe no longer rolled up completely into a single vortex ring. Instead, a leading ring formed and a trail of vorticity followed behind. With the increasing of the ratio $L / D$, the leading vortex ring no longer grows in strength but the trail getting stronger, suggesting that only a certain fraction of the vorticity created at the opening during the ejection can be entrained to form the leading vortex ring for large stroke ratios. In squid jet 
propulsion, the jet flow structure and propulsive efficiency are dependent upon the formation number as well.

\section{Mathematics and Numerical Models}

\subsection{Geometrical Model Setup of Squid Mantle Contraction}

The simplified numerical model (Figure 2) in this paper is described in dimensional form to facilitate comparison with previous experiments carried out by Joseph T. Thompson and Erik J. Anderson [17]. It is a round-tailed axisymmetric body with a cone-shaped funnel and a columnar jet tube. Other body characters of squid such as head and arms are ignored. The model has an outer diameter of 80 $\mathrm{mm}$. and a length of $270 \mathrm{~mm}$. The length of the mantle is $180 \mathrm{~mm}$ and the initial thickness of the mantle is $4 \mathrm{~mm}$. To do corresponding contrastive analysis on the model parameters, the length and the inner diameter of the columnar jet tube are alterable, signed as $L n$ and $D$. Figure 2 shows the meridian cross-section section of the model. In the present Figureures $L n=38 \mathrm{~mm}$ and $D=24 \mathrm{~mm}$. To simplify the contraction and inflation motion of the squid mantle, the outside wall of the mantle is supposed to stay fixedly, the inside wall of the mantle moves up and down with a given velocity, $v$. This means that the thickness of the squid mantle changed with time. The movement of the inside wall of the mantle is defined by velocity and time based on Erik J. Anderson's research [1-17] on living squid. When the mantle contract, $v$ lasts $0.6 \mathrm{~s}$ and when the mantle inflate $v$ lasts $1 \mathrm{~s}$.

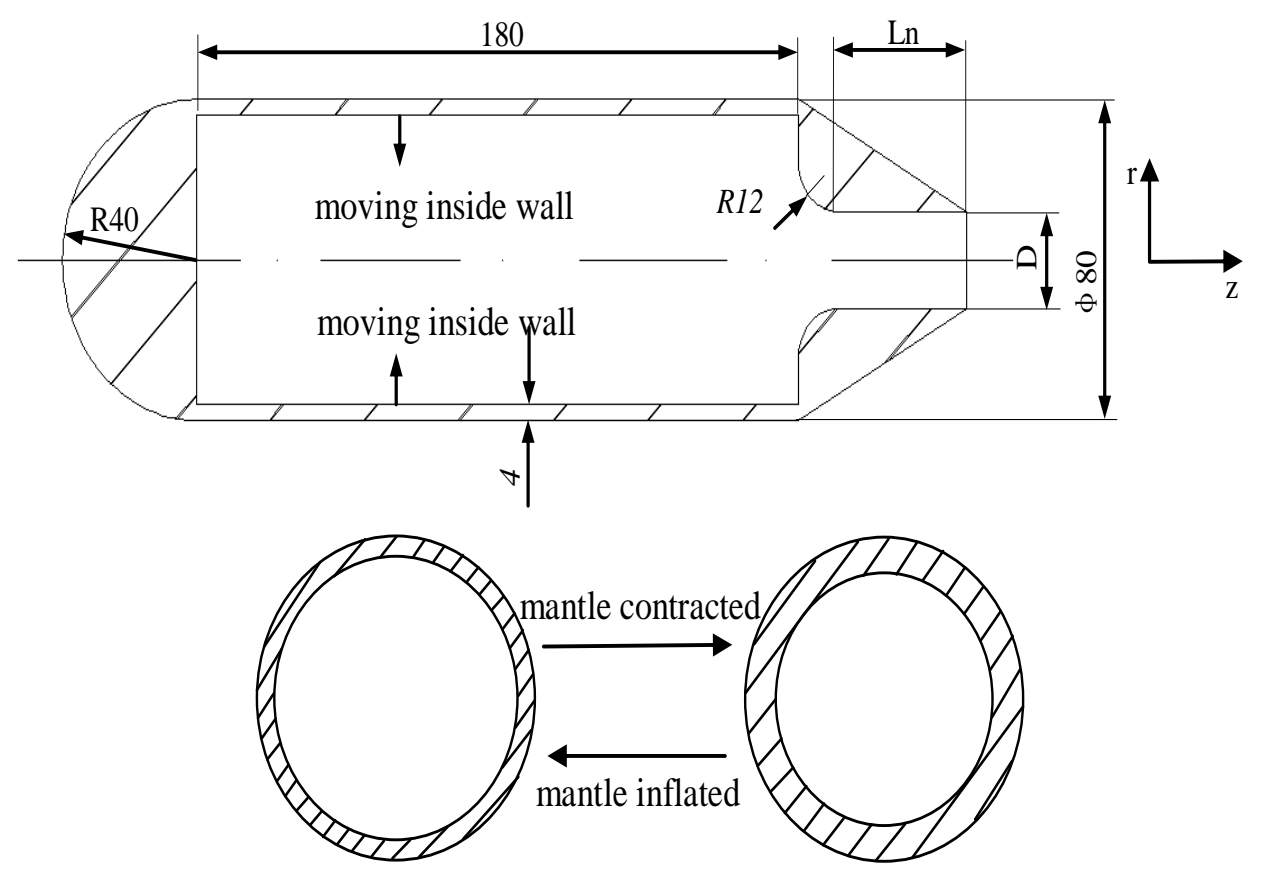

Figure 2. The Meridian Cross-Section Section and the Mantle Movement the Model

\subsection{Calculations of Jet Length-to-Diameter Ratio (L/D)}

In those piston jetting models, the length of the plug of fluid expelled from the pipe is supposed approximately equal to the displacement of the piston. But in this paper, a mantle contracted model has been set up. So the jet length-to-diameter ratio $L / D$ is calculated to find out the interrelated influencing factors on it. 
Some hypotheses were proposed firstly: the density of water is a constant; the water is inviscid fluid; the jet velocity at the nozzle section is identical; the shape of the fluid has been emitted out retain as a pipe. Figure 3 shows the ideal mathematic model for mantle contracted jetting with the interrelated parameters.

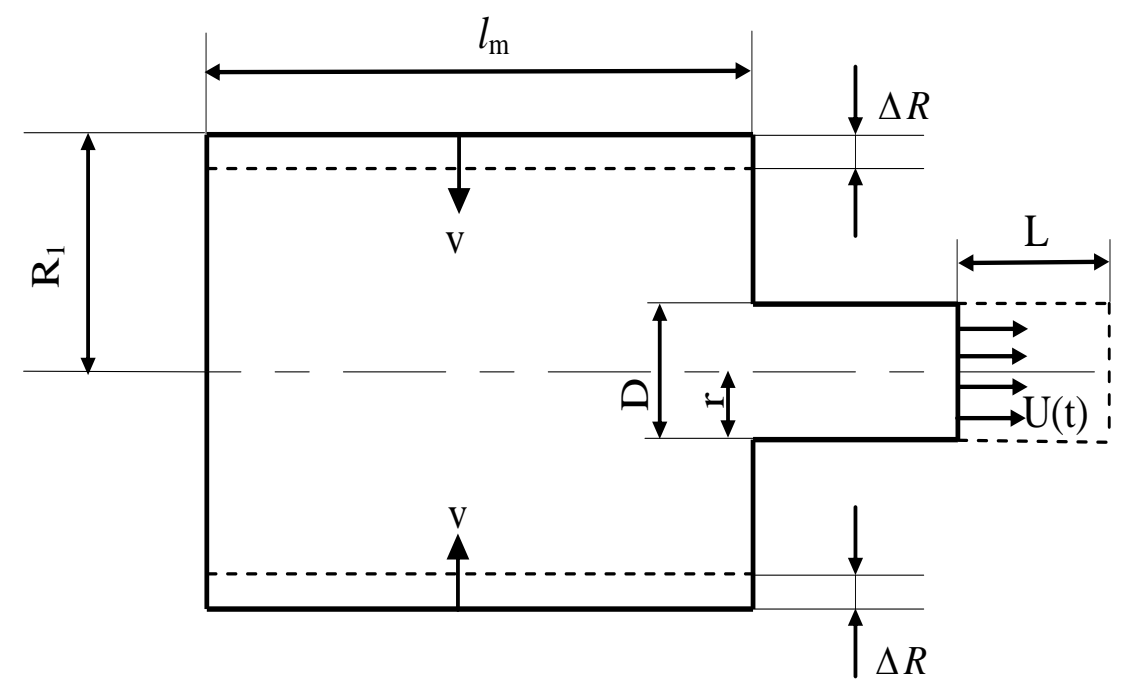

Figure 3. The Ideal Mathematic Model for Mantle Contracted Jetting with the Interrelated Parameter

Then the following equations can be gained from Figure 8:

$d V_{1}(t)=2 \pi R(t) R^{\prime}(t) d t \times l_{m}$

$R(t)=R_{1}-v t$

$d V_{2}(t)=\pi r^{2} U(t) d t$

$d V_{1}(t)=d V_{2}(t)$

Using equations (1) - (5), the jet velocity $U(t)$ at time $t$ can be obtained:

$U(t)=\frac{2\left(R_{1}-v t\right) \times v \times l_{m}}{r^{2}}$

Where $\mathrm{R}_{1}$ and $\mathrm{lm}$ are the initial radius and length of squid mantle, $v$ is the contraction velocity of squid mantle, $R(t)$ is the radius for every time $t, r$ is the radius of the nozzle. Then the formation number of the vortex ring is:

$L / D=\frac{\int_{0}^{t} U(t) d t}{2 r}=\frac{l_{m} v}{r^{3}} \int_{0}^{t}\left(R_{1}-v t\right) d t=\frac{l_{m} v t\left(R_{1}-\frac{1}{2} v t\right)}{r^{3}}$

From equation (6), if the initial radius $R_{1}$, the length of squid mantle $l_{m}$ and time $t$ is fixed, the formation number $L / D$ lies on the mantle contraction velocity $v$ and the radius of the nozzle $r$.

\subsection{Governing Equations}

Assuming that the flow is unstable, laminar, incompressible, and Newtonian flow. Further, the flow is assumed to be axisymmetric structure. Therefore, the control 
equation is axisymmetric unsteady incompressible Navier-Stokes equations and the continuity equation.

Throughout the present numerical simulations, the fluid density, $\rho$ is $998.2 \mathrm{~kg} / \mathrm{m}^{3}$ and the fluid kinematic viscosity, $\mu$ is $1.0 \times 10^{-6} \mathrm{~m}^{2} / \mathrm{s}$. The governing equations is described in circular cylindrical coordinates $(r, \theta, z), r$ is the radial direction and $z$ is the axis direction. $u_{i}(i=r, \theta, z)$ is the velocity component in three directions. $p$ is the pressure.

Navier-Stokes equations:

$$
\begin{aligned}
& \frac{\partial u_{r}}{\partial t}+u_{r} \frac{\partial u_{r}}{\partial r}+u_{z} \frac{\partial u_{r}}{\partial z}-\frac{u_{\theta}^{2}}{r}+\frac{1}{\rho} \frac{\partial p}{\partial r}=\mu\left(\nabla^{2} u_{r}-\frac{u_{r}}{r^{2}}\right) \\
& \frac{\partial u_{\theta}}{\partial t}+u_{r} \frac{\partial u_{\theta}}{\partial r}+u_{z} \frac{\partial u_{\theta}}{\partial z}+\frac{u_{r} u_{\theta}}{r}=\mu\left(\nabla^{2} u_{\theta}-\frac{u_{\theta}}{r^{2}}\right) \\
& \frac{\partial u_{z}}{\partial t}+u_{r} \frac{\partial u_{z}}{\partial r}+u_{z} \frac{\partial u_{z}}{\partial z}+\frac{1}{\rho} \frac{\partial p}{\partial z}=\mu \nabla^{2} u_{z}
\end{aligned}
$$

Continuity equation:

$\frac{\partial\left(r u_{r}\right)}{\partial_{r}}+\frac{\partial\left(r u_{z}\right)}{\partial_{z}}=0$

Where

$$
\nabla^{2}=\frac{\partial^{2}}{\partial r^{2}}+\frac{1}{r} \frac{\partial}{\partial r}+\frac{\partial^{2}}{\partial z^{2}}
$$

\subsection{Mesh Structure and Moving Mesh}

Mesh construction is important as both the accuracy of solution and the subsequent calculation time are dependent on the fineness of the grid. An axisymmetric setup is used in the model because two-dimensional flow features dominate the flow. Assuming axisymmetry can also reduces computational costs greatly. The size of the computational domain is $1520 \mathrm{~mm}$ in the axial, $\mathrm{z}$, direction, and $200 \mathrm{~mm}$ in the radial, $\mathrm{r}$, direction(Figure 4). The domain is divided into several parts and each of them contains a structured grid. The calculational setup is modeled and meshed in Gambit, a software for preprocess.

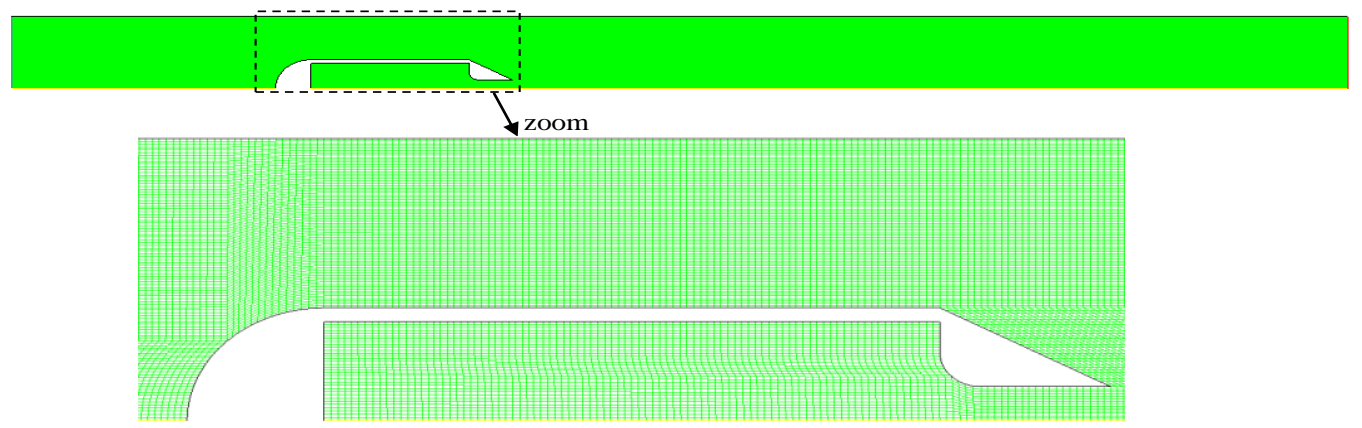

Figure 4. Grid Distribution Around Squid Model 
The inside wall of the mantle is defined as a moving boundary. Because the moving boundary is a purely linear motion and the adjacent mesh elements are quad meshed, the dynamic model makes use of dynamic layering as its meshing scheme. The numerical algorithm automatically updates the mesh after each time step relative to the mantle's motion using dynamic layering. The dynamic layering scheme adds or removes layers of cells adjacent to a moving boundary based on the height of the layer adjacent to the moving surface.This model uses Fluent userdefined function (UDF)described mantle movement.

\section{Results and Discussion}

\subsection{Vortex Ring Formation During Jetting of Squid}

Define the mantle contract velocity, $v=0.006 \mathrm{~m} / \mathrm{s}$, lasts $0.6 \mathrm{~s}$ and the mantle inflate velocity, $v=-0.0036 \mathrm{~m} / \mathrm{s}$, lasts $1 \mathrm{~s}$. The results of the current simulation show a vortex ring model to analyze the hydrodynamics of squid jet propulsion. Figure 5 shows that some individual torroidal fluid structures will be shed from the trailing edge of the jet nozzle during squid jet process. These torroidal flows are vortex rings. They appear in cross-section as two counter-rotating vortices. Flow at the center of an emitted vortex ring is in the direction of the original emission of fluid from the jet nozzle.

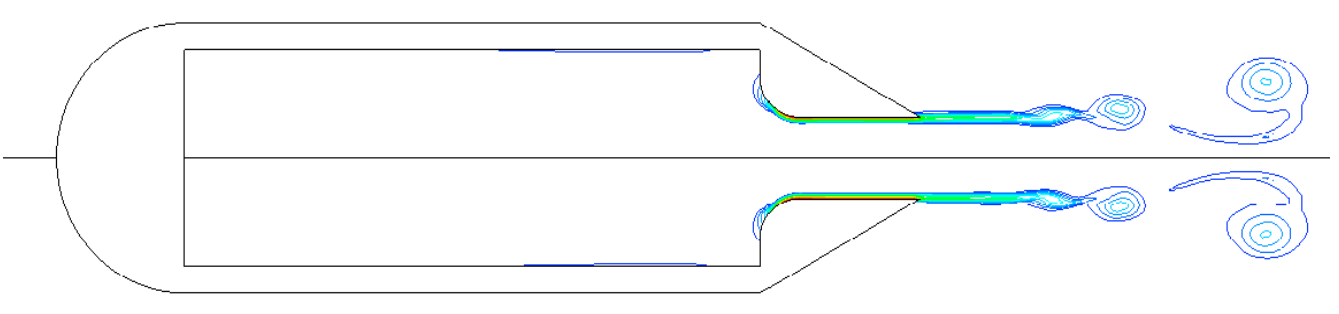

Figure 5. A Vortex Ring Model

Figure 6 shows the formation and evolution of a vortex ring shed from the jet nozzle during the mantle contract process. At the beginning of jet process, a boundary layer flow occurs on the solid wall when the fluid flows through the jet nozzle at a speed. Across the boundary layer the flow velocity changes from the value zero at the boundary to some finite value characteristic of an inviscid fluid. So within the boundary it is full of vorticity and outside the boundary the vorticity is zero. The vorticity is then diffused away by viscosity and convected downstream with the fluid. Then the boundary layer separates from the jet nozzle because the velocity of the effectively inviscid fluid just outside the boundary layer decreases in the flow directions rapidly. The shed vortices roll up into three-dimensional (3D) ring-like structures in the still water. The flow field consists of a leading vortex ring followed by a trailing jet. The size of the leading vortex ring becomes bigger during the jet process, and when the jetting process stops, the size of the leading vortex ring stays at a certain size and pinches off from the vorticity tail behind it. 


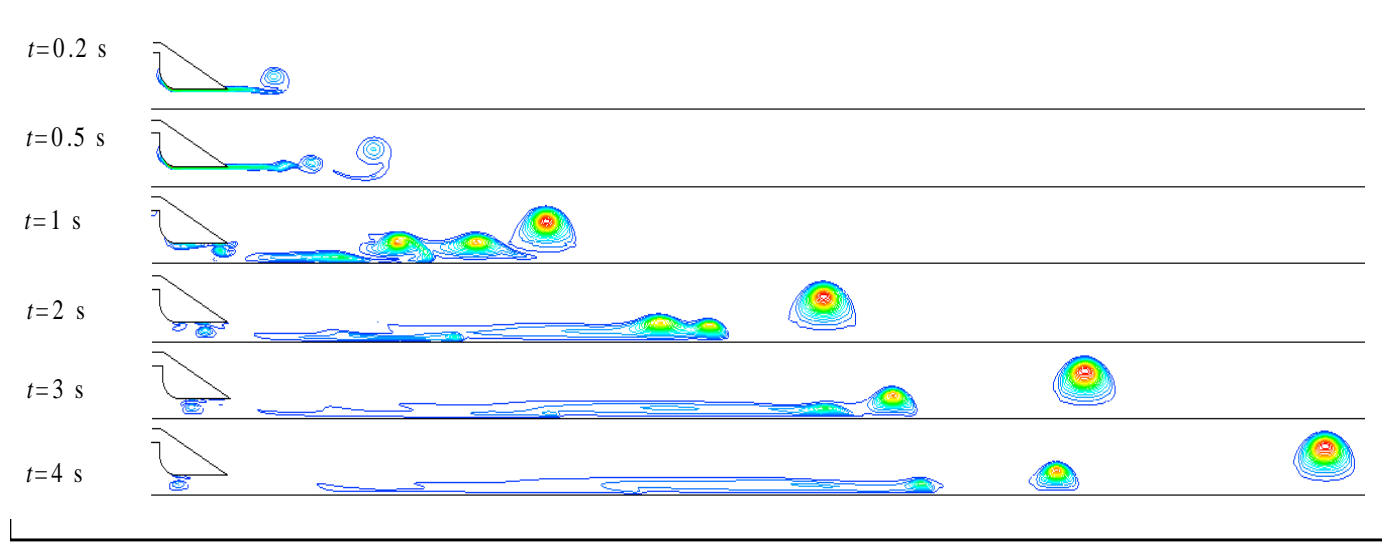

Figure 6. The Formation and Evolution of Vortex Ring

\subsection{Different Vortex Rings under the Influence of Formation Number}

Based on the model, simulations have been done by just changing the mantle contraction velocity $v$ or the radius of the nozzle $r$ to observe the different formations of vortex ring. Figure 7 shows different vortex rings under different mantle contraction velocities at the time 2 second after the jetting starts. Using equation (6), when the mantle contraction velocity $v=0.002 \mathrm{~m} / \mathrm{s}$, the formation number is 1.97. A single vortex ring should be formed by the forward conclusion and it is proved in the simulation (Figure. 9). When the mantle contraction velocity $v=0.004 \mathrm{~m} / \mathrm{s}$, the formation number computed by equation (6) is 3.7 , close to the critical formation number. But there already exits a trail of vorticity in the simulation which conflicts with the conclusion in part 2. The reason of this phenomenon is mainly ignoring of the viscosity of water in equation (6). The actual formation number is bigger than the calculate quantity, 3.7. Along with the increase of the mantle contraction velocity from $0.006 \mathrm{~m} / \mathrm{s}$ to $0.01 \mathrm{~m} / \mathrm{s}$, the strength and size of the trail of vorticity increases accordingly but the leading vortex ring remains almost the same size.

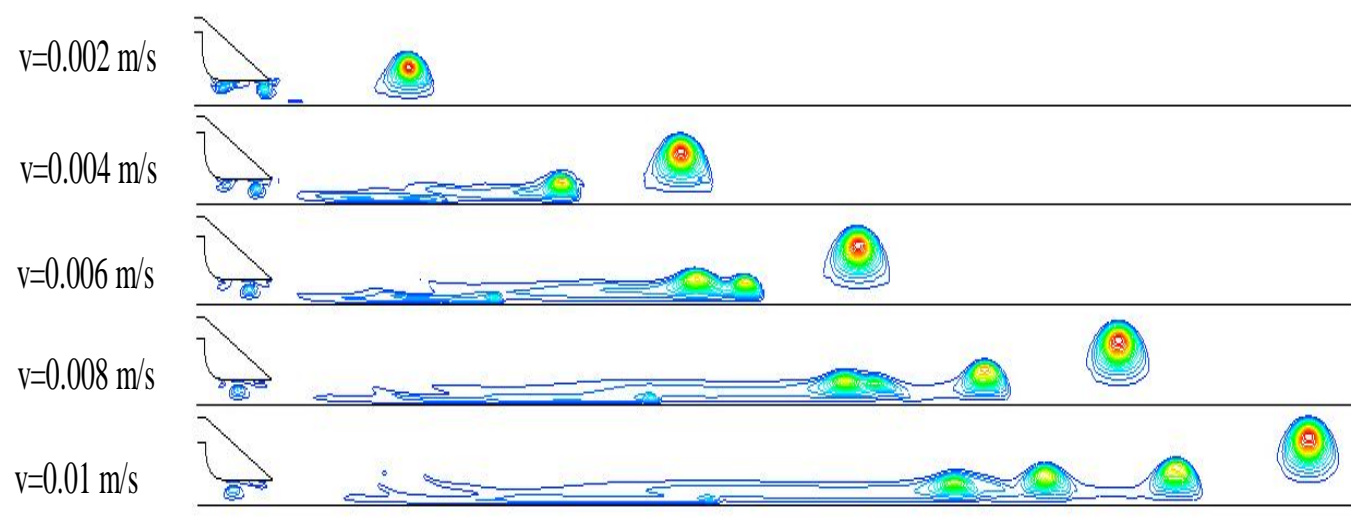

\section{Figure 7. Vortex Ring Formation Under Different Mantle Contraction Velocities}

When the formation number is approximately 4, a maximum single vortex ring will be formed in still water during the jet process. This is an optimal vortex ring because the corresponding propulsive efficiency becomes highest. The vortex with $L / D=4$ has the maximum momentum for a given energy input, i.e. it will produce the maximum thrust on the body generating it. When the $L / D$ is greater than 4 , vortex 
ring no longer grows in strength but rather 'pinches off' from the jet, and the remaining fluid in the pulse is simply ejected as a trailing jet. This 'pinches off' phenomenon also has significance for propulsion since it is shown that the formation of a vortex ring generates proportionally more impulse per unit of ejected fluid than does a trailing jet. The propulsive efficiency decreases as the increase of the formation number $L / D$. But hydrodynamic efficiency may be the major factor that led to the evolution of this mechanism of propulsion in squid. Rapid acceleration to achieve the maximum speed during escape may be more important for their survival than the 'efficiency' during sustained swimming. Squid usually produced jets with large $L / D$. Anderson [17] studied on an adult live squid whose size is $27.1 \pm 3.1 \mathrm{~cm}$ (mean \pm S.D.), which is the same as the model in the present paper. He also found that the corresponding $L / D$ is approximately 40 . The conclusion coincided with the vorticity distribution simulated in the Figure6 quite well.

Figure. 8 shows that the change of the nozzle diameter from $12 \mathrm{~mm}$ to $28 \mathrm{~mm}$ significantly influences the vorticity distribution. The different vortex rings are at the time 0.6 second after the jetting starts. As the decrease of the nozzle diameter, the length of the trail of vorticity increases more and more fast, so the $L / D$ increases fast and the jet propulsive efficiency decrease fast. Figure. 9 shows two squids and their funnels. Through the squid dissection and measurement, the mantle of the bigger one has a length of $327 \mathrm{~mm}$ and an outer diameter of $93 \mathrm{~mm}$, the funnel of the bigger one has a inner diameter of $9.6 \mathrm{~mm}$. The mantle of the smaller one has a length of $267 \mathrm{~mm}$ and an outer diameter of $70 \mathrm{~mm}$, the funnel of the bigger one has a inner diameter of $8.9 \mathrm{~mm}$. The ratio of the nozzle diameter and the mantle diameter of smaller squid is 0.13 , lager than the ratio of bigger squid, 0.1 , so smaller squid have larger relative funnel diameters. Smaller squid have lower $L / D$ ratios and can achieve higher propulsive efficiency by jetting. Further more, smaller squid have limited strength, they endure lager viscous force, so they have to increase their swimming efficiency to save energy.

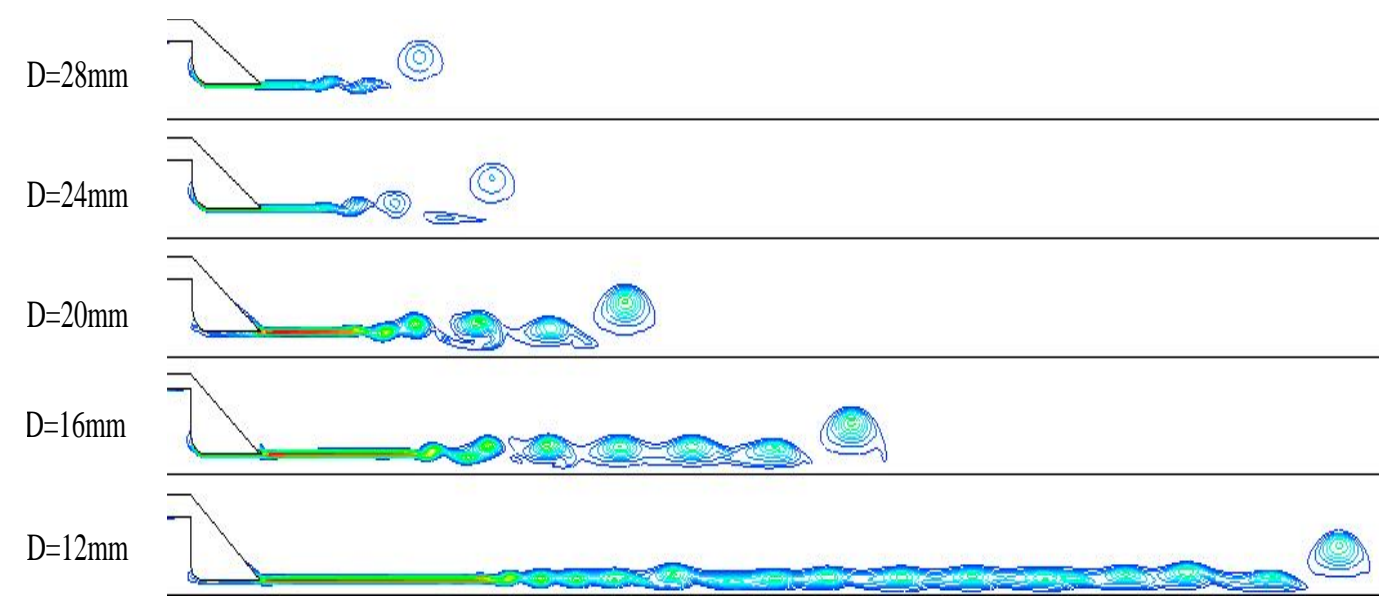

Figure 8. Vortex Rings Formation Under Different Nozzle Diameters 


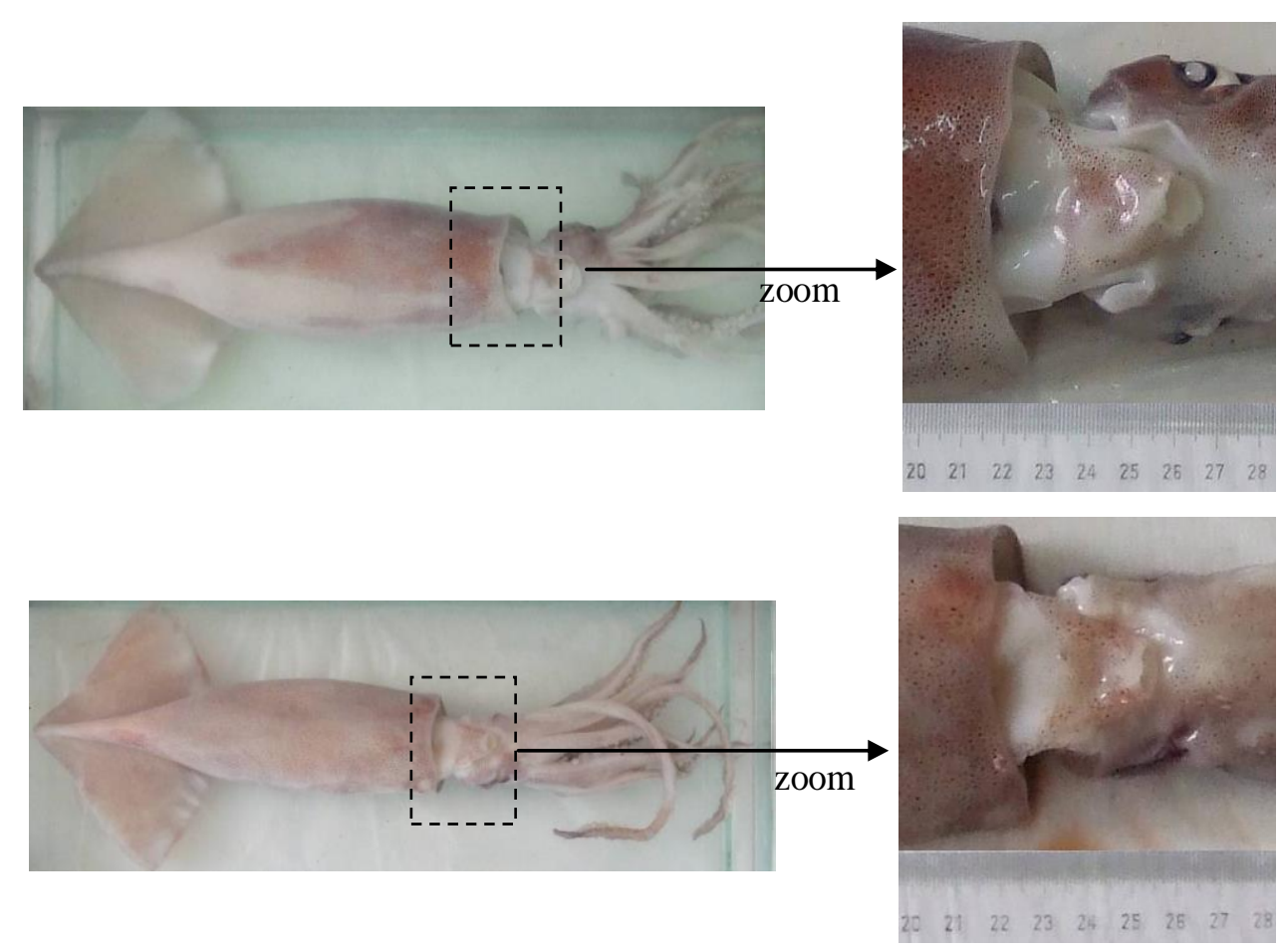

Figure 9. The Squid Dissection and Measurement

To investigate whether the nozzle length influence the vorticity distribution, numerical simulations have been carried out with changing the nozzle length from $12 \mathrm{~mm}$ to $120 \mathrm{~mm}$. The vortex rings are at the time 3 second after the jetting starts. The result (Figure.10) shows that few differences of the formation of the vortex ring exit in the five conditions, which means the nozzle length has few influence on the vortex evolution behavior.

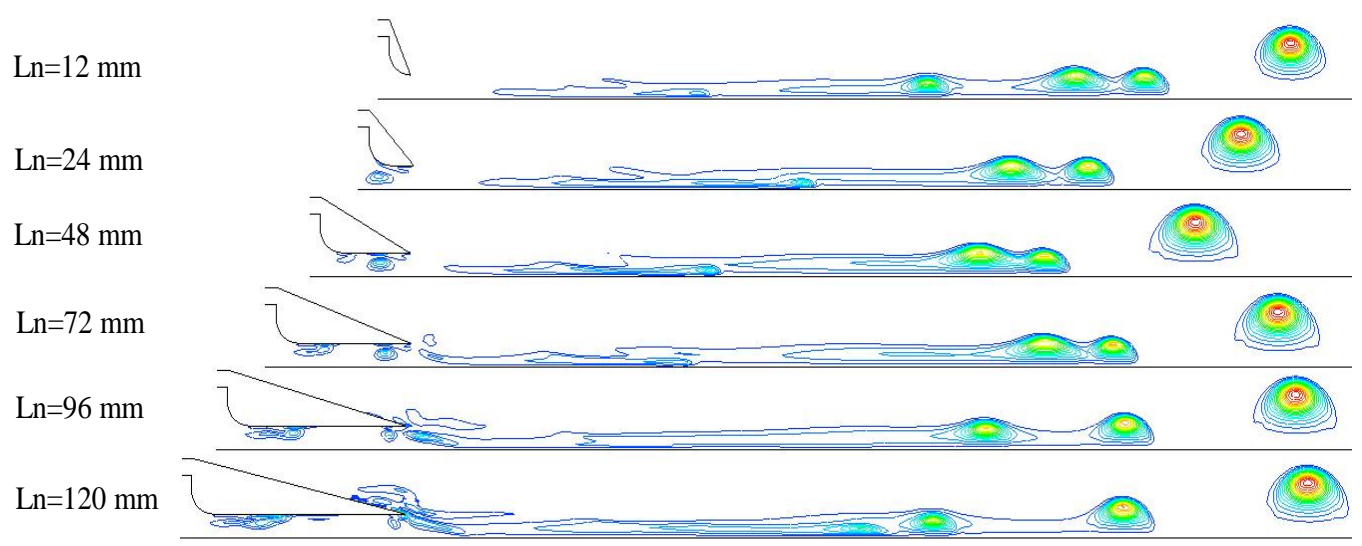

Figure 10. Vortex Rings Formation Under Different Nozzle Lengths

\subsection{Interaction of Vortex Rings formed in Two Jet Processes}

Research by Bartol I. K. [18] shows that squid will increase their mantle contraction frequency to elevate swimming speed. This means that thrust may be improved by simply keeping the spacing between vortex rings low by increasing contraction frequency may improve thrust. Squid may benefit from jetting water at a high frequency by interacting vortex rings, which can produce a greater overall translational velocity. 
Two periods of the mantle movement has been simulated. Define the mantle contraction velocity, $v=0.006 \mathrm{~m} / \mathrm{s}$, lasts $0.6 \mathrm{~s}$ and the mantle inflation velocity, $v$ $=0.0036 \mathrm{~m} / \mathrm{s}$, lasts $1 \mathrm{~s}$. The result (Figure 11) shows that the leading vortex ring formed in the second jet process move with a higher velocity than the trail formed in the first jet process. The leading vortex ring would catch the front trail up at a time. Then they will interact with each other and a new stronger vortex ring formed. In the process, more water might be rolled into the new vortex ring. The new vortex ring can increase the amount of fluid driven backwards in the jet wake. Based on the law of momentum conservation the jet thrust will increase, so squid propulsion can benefit from the interaction.

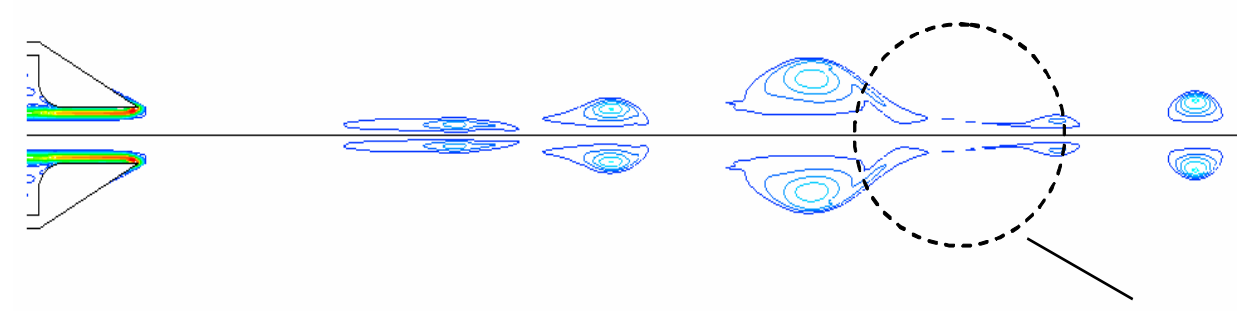

The interacting zone

\section{Figure 11. Interaction of Vortex Rings Formed in Two Jet Processes}

\subsection{Simulation Results of Thrust Force}

The thrust force was also simulated during the jetting process on the model. Figure 12 shows the unsteady force changes during the action of the mantle inner wall action in $0.6 \mathrm{~s}, \mathrm{v}=0.006 \mathrm{~m} / \mathrm{s}, \mathrm{D}=24 \mathrm{~mm}$. The propulsive force experiences a rapid increase to a maximum value first, then slowly shrinking process, the peak size of $113.8 \mathrm{mN}$. Simulation results give the thrust force change under a range of mantle contract velocity. The force increases fast as the mantle contract velocity. The curve like a parabola(Figure 13).

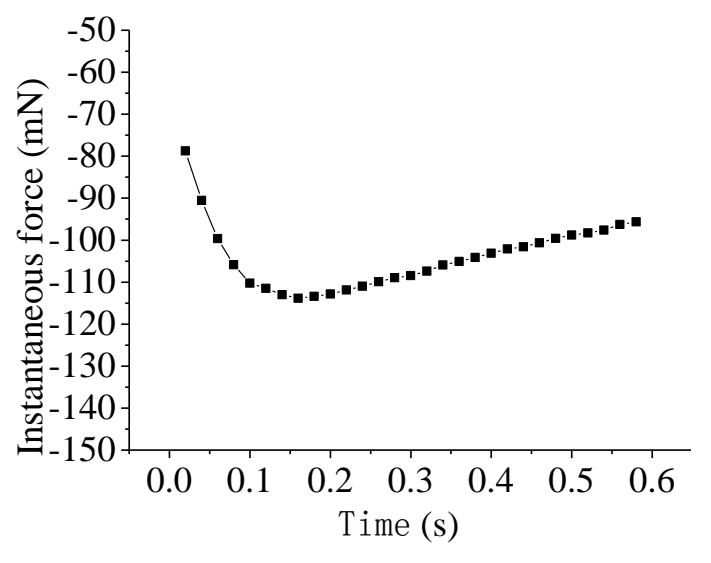

Figure 12. Simulation Results of
Thrust Force

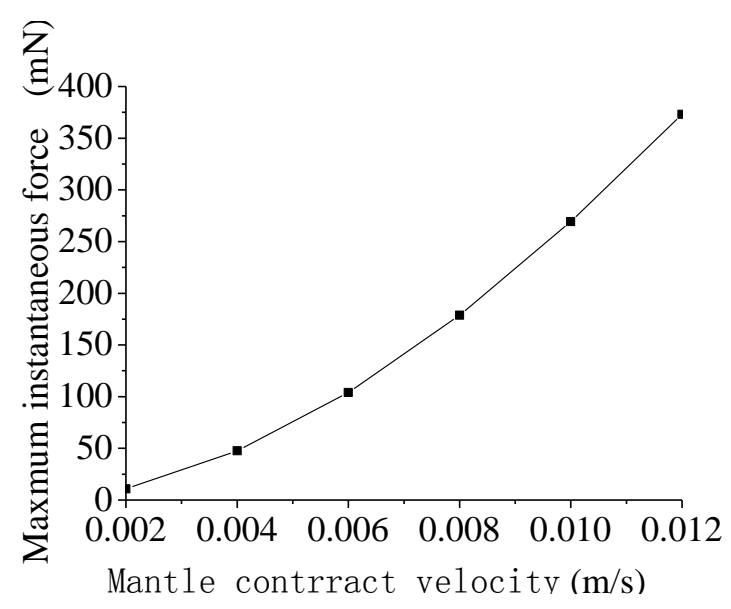

Figure 13. Simulation Results of Thrust Force under a Range of Mantle Contract Velocity 


\section{Experiment on a Bionic Jet Propulsor}

To validate the simulation results of thrust force, experiments on a bionic jet propulsor have benn performed. Imitating the muscle structure of squid, a biomimetic water-jetting propulsor actuated by SMA (shape memory alloy)wires has been investigated. The propulsor consists of the biomimetic mantle and funnel (Figure 14). The cross-section of the biomimetic mantle is circular. The foreside and the circle side of the biomimetic mantle are closed while the rearward is open. The refill and jet can be achieved by diametrical change of the biomimetic mantle. Funnel is installed at the rearward openings of the mantle. The back-end of the funnel is the jet orifice. The elastic energy resilience mechanism is employed in the mantle movement process. When the SMA wires are electrical heated, they contract because of their inner phase transformation. The forces generated by SMA wires along the ribs direction can be counteracted because they are in opposite direction. Force generated on the SMA wire can reduce the vertical distance between adjacent libs. This will cause an increase in biomimetic mantle thickness. Because the volume of bionic mantle is substantially constant, then inevitably lead to reduced bionic mantle diameter, while the elastic energy stored in the silicon gel. When the electric heating end, SMA wire began to change into martensite phase. The stored energy is released in the silicon gel, driving SMA wire returns to its original length. Silicone gel is also returned to its initial thickness. It will lead to increase in diameter bionic mantle. This process again and again. Pulse jet propulsion SMA wires through heating and cooling cycles to achieve.
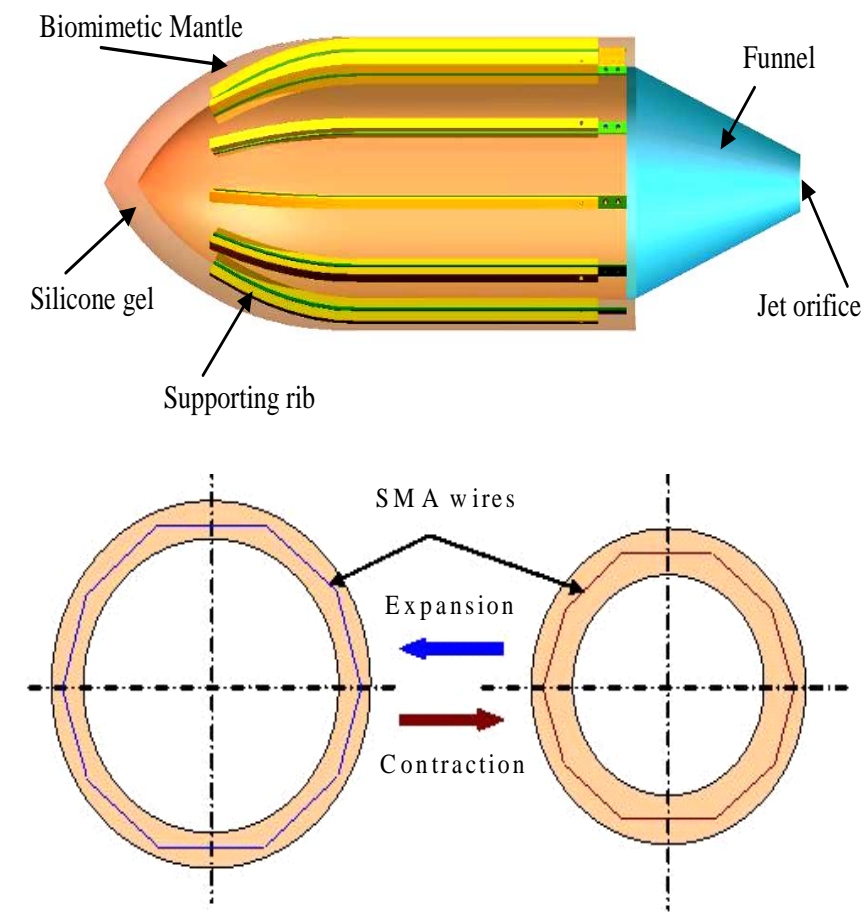

\section{Figure 14. The Structure of the Biomimetic Water-Jetting Propulsion Vehicle and the Action Process}

In order to test the performance of the bionic jetting propulsor, thrust testing experiment was done. The vehicle mantle has a length of $260 \mathrm{~mm}$, an outside diameter of $110 \mathrm{~mm}$, an inside diameter of $86 \mathrm{~mm}$ and 11 supporting ribs. The diameter of the SMA wires is $0.175 \mathrm{~mm}$. The funnel of PVC has a length of $100 \mathrm{~mm}$ and a thickness of $0.2 \mathrm{~mm}$. The jet orifice diameter is $23 \mathrm{~mm}$. The bionic jetting 
propulsor was heated by pulse eclectic current. Digital video recordings of the motion of the biomimetic vehicle were used to determine very accurate biomimetic mantle deformations. Figure 15 shows the section change under electric current 30 $\mathrm{A}$ and heating time is $1.6 \mathrm{~s}$. The contraction rate of the section area is $15.4 \%$. At the same time, the thrust is recorded by force sensors.

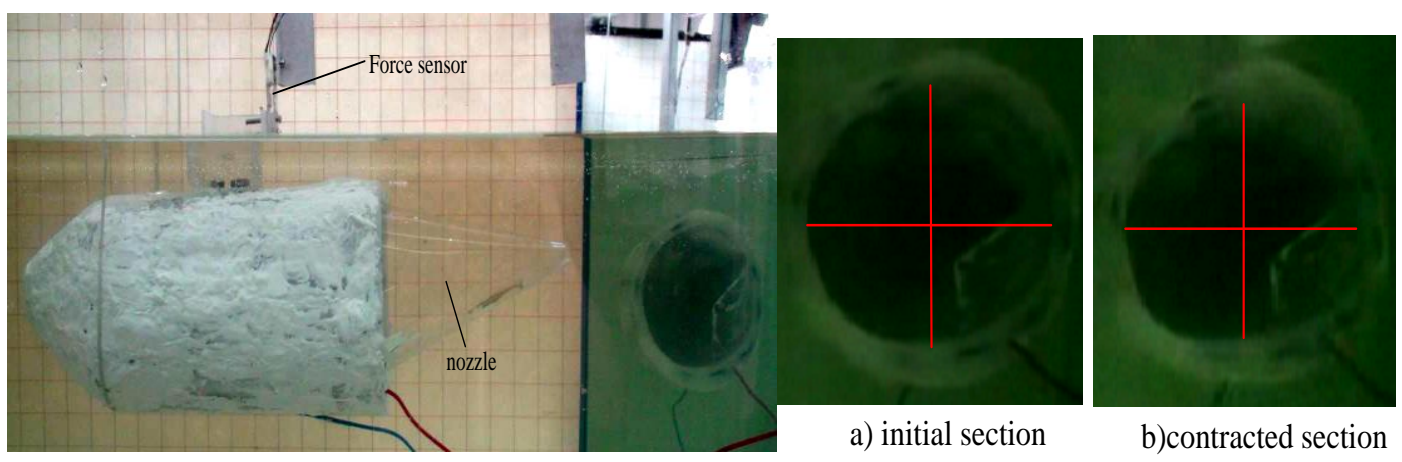

Figure 15. Experiment Setup on Pulse Jet Propulsor and its Section Change

Figure 16 shows the instantaneous force curve over time during the action of the bioinc mantle.It can be seen that when the mantle starts operation, propulsive force generated increases rapidly, then although the mantle is still in the contraction process, the propulsive force began a slow decrease. The propulsive force reduces to 0 at the end of the process. In the experiment, the relation of voltage and propulsive performance has been studied.The pulse width is fixed at $1600 \mathrm{~ms}$ and the supply voltage varies from $18 \mathrm{~V}$ to $30 \mathrm{~V}$. Figure 17 shows the change of maximum instantaneous thrust under different power-voltage. Similar with the curve in figure 13 well, the maximum instantaneous thrust rapidly increases first and decreases slowly then.

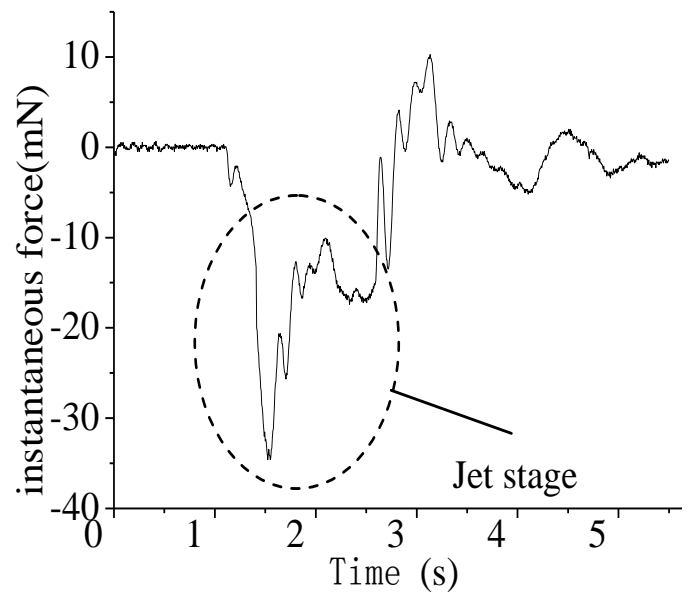

Figure 16. Experimental Results of Thrust Force

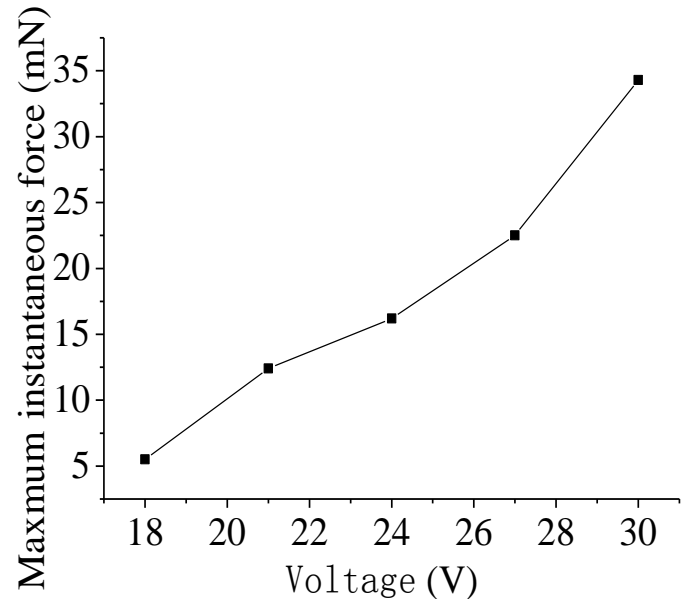

Figure 17. Experimental Results of Thrust Force Under a Range of Voltages

\section{Conclusions}

In this study, numerical simulations were carried out to examine the vortex ring model and to investigate its effect in squid jet propulsion. Vortex ring formation without background flow was simulated in the squid jet propulsion. The propulsive 
efficiency of squid is related with the formation of the vortex ring which is dependent upon the formation number $L / D$. In the present setup, the mantle contracted velocity and the nozzle diameter are the main factors. After simulations with different parameters, different vortex evolution behavior was observed. When the mantle contracted velocity is small enough of the nozzle diameter is large enough, the formation number $L / D<4$, a single vortex ring would be formed in the jet process. In contrast, when the formation number $L / D>4$, a leading ring formed and a trail of vorticity followed behind in the jet process. As the increase of the formation number $L / D$, the propulsive efficiency decrease. Those jet mechanics can provide academic basis for the bionic underwater robots which propelled themselves by jetting water like squid. The numerical simulation results prove: (1) the formation number of squid jet propulsion is usually a large number, so the hydrodynamic efficiency may not be the major factor that led to the evolution of this mechanism of propulsion in squid; (2) smaller squid have larger relative funnel diameters to achieve relatively higher propulsive efficiency; (3) squid expel water at high frequency may get benefit because vortices are able to interact. Anyway, using CFD to simulate the flow structure in squid jet propulsion is a viable and powerful problems-solving tool in biomimetics.Moreover, the thrust force experiment results on a bionic jet peopulsor accord with the simulated force results well.

For future work, the background flow should be considered in this model, as there might be interaction between the background flow and the jet flow.

\section{Acknowledgments}

This work is funded by China Postdoctoral Science Foundation (Grant No.2015M571382), Natural Science Foundation of Heilongjiang Province of China(Grant No.QC2013C058).

\section{References}

[1] E. J.Anderson and M. E. Demont, "The mechanics of locomotion in the squid loligo pealei: locomotory function and unsteady hydrodynamics of the jet and intramantle pressure", The Journal of Experimental Biology, vol. 203, (2000), pp. 2851-2863.

[2] J. T.Thompson and W. M.Kier, "Ontogenetic Changes in Mantle Kinematics During Escape-Jet Locomotion in the Oval Squid", Sepioteuthis lessoniana Lesson, 1830.The Biological Bulletin, vol. 201, (2001), pp. 154-166.

[3] J. T. Thompson and W. M.Kier, "Ontogeny of Squid Mantle Function: Changes in the Mechanics of Escape-Jet Locomotion in the Oval Squid", Sepioteuthis lessoniana Lesson, 1830, The Biological Bulletin, vol. 203, (2002), pp. 14-26.

[4] H. Jiang and M. A, "Grosenbaugh, "Numerical simulation of vortex ring formation in the presence of background flow with implications for squid propulsion", Theor. Comput.Fluid Dyn, vol. 20, no. 2, (2006), pp. 103-123.

[5] I. K. Bartol., "Role of Aerobic and Anaerobic Circular Mantle Muscle Fibers in Swimming Squid: Electromyography", Biol. Bull., vol. 200, (2001), pp. 59-66.

[6] K.Shariff and A.Leonard, “Vortex rings”, Ann. Rev. Fluid Mech.,vol. 34, (1992), pp. 235-279.

[7] E. G. Drucker and G. V. Lauder, "Locomotor forces on a swimming fish: three-dimensional vortex wake dynamics quantified using digital particle image velocimetry. J. Exp. Biol., no. 202, (1999), pp. 2393-2412.

[8] Q.Bone and E. R. Trueman, "Jet propulsion in salps (Tunicata: Thaliacea)", J. Zool. Lond, no. 201, (1983), pp. 481-506.

[9] L. P. Madin, “Aspects of jet propulsion in salps", Can. J.Zool., no. 68, (1990), pp. 765-777.

[10] T. L. Daniel ,"Mechanics and energetics of medusan jet propulsion", Can. J. Zool., vol. 61, (1983), pp. $1406-1420$.

[11] P. S.Krueger and M.Gharib, "The significance of vortex ring formation to the thrust and impulse of a starting jet", Phys. Fluids, vol. 15, (2003), pp. 1271-1281.

[12] P. F. Linden and J. S. Turner " "Optimal vortex rings and aquatic propulsion mechanisms", Proc. R. Soc. Lond. B, vol. 271, (2004), pp. 647-653.

[13] P. F. Linden and J. S. Turner, " The formation of 'optimal' vortex rings, and the efficiency of propulsion devices”, J. Fluid Mech., vol. 427, (2001), pp. 61-72. 
[14] M. Gharib, E. Rambod and K. Shariff, "A universal time scale for vortex ring formation”, Journal of Fluid Mech., no. 360, (1998), pp. 121-140.

[15] J. O. Dabiri and M.Gharib, "Fluid entrainment by isolated vortex rings", Journal of Fluid Mech., no. 511, (2004), pp. 311-331.

[16] P. S. Krueger, J. O.Dabiri and M.Gharib, "Vortex ring pinch off in the presence of simultaneously initiated uniform background co-flow", Phys. Fluids, vol. 15, (2003), pp. L49-L52.

[17] E. J. Anderson and M. A. Grosenbaugh, "Jet flow in steadily swimming adult squid", The Journal of Experimental Biology, vol. 208, (2005), pp. 1125-1146.

[18] I. K. Bartol, M. R. Patterson and R. Mann, "Swimming mechanics and behavior of the shallow-water brief squid Lolliguncula brevis”, The Journal of Experimental Biology, vol. 204, (2001),pp. 36-55. 
International Journal of $u-$ and e- Service, Science and Technology Vol.9, No. 5 (2016) 